\title{
Design of nanofibres for efficient stimulated Raman scattering in the evanescent field
}

\section{Shan \\ G. Pauliat \\ G. Vienne}

\begin{abstract}
Laboratoire Charles Fabry, Institut d'Optique, CNRS, Univ Paris-Sud, 2 av. A. Fresnel, 91127 Palaiseau cedex, France

Laboratoire Charles Fabry, Institut d'Optique, CNRS, Univ Paris-Sud, 2 av. A. Fresnel, 91127 Palaiseau cedex, France

State Key Laboratory of Modern Optical Instrumentation, Department of Optical Engineering, Zhejiang University, Hangzhou 310027, China

Present address: Data Storage Institute, Agency for Science, Technology and Research, (A*STAR), Singapore 117608 , China
\end{abstract}

L. Tong

State Key Laboratory of Modern Optical Instrumentation, Department of Optical Engineering, Zhejiang University, Hangzhou 310027, China

Laboratoire Charles Fabry, Institut d'Optique, CNRS, Univ Paris-Sud, 2 av. A. Fresnel, 91127 Palaiseau cedex, France
S. Lebrun

sylvie.lebrun@institutoptique.fr
Nanofibres can be produced with diameters smaller than the wavelength of the light they guide. In this regime, the guided mode presents a strong evanescent field well adapted to the excitation of "evanescent nonlinearities". We theoretically investigate such an evanescent nonlinearity: the Raman interaction between the evanescent field and a liquid surrounding the nanofibre. Our calculations demonstrate that the Raman conversion is obtained with nanofibre lengths an order of magnitude lower than those required for liquid core photonic crystal fibres. [DOI: http://dx.doi.org/10.2971/jeos.2013.13030]

Keywords: Fibre design and fabrication, nonlinear optics, optical fibres, Raman effect

\section{INTRODUCTION}

Tapered nanofibres are produced by stretching conventional fibres, typically telecom fibres, until their diameter reaches a few hundred nanometres [1, 2]. During its propagation in the tapered section, the mode guided by the core of the initial un-tapered fibre is progressively transformed into a mode guided by the nanofibre. The light confinement as the fibre diameter is reduced enhances the intensity up to a few hundred times. Considering that nanofibres can be pulled over tens of centimetres, one anticipates advantages in using nanofibres instead of conventional fibres to perform nonlinear experiments. This feature did not escape the attention of other research teams and was underlined in the literature [3]. Several nonlinear effects have already been investigated:

- Supercontinuum generation, either in the femtosecond regime or in the sub-nanosecond regime [2]-[10];

- Second and third harmonic generation [11]-[13].

When the nanofibre diameter becomes comparable or smaller than the wavelength, the guided light exhibits a pronounced evanescent field that envelops the fibre [1]. In the abovementioned experiments, this evanescent field was mainly used to manage the dispersion. Exploiting this large evanescent field to implement sensors or atomic fluorescence probes has also already been suggested [14]-[17]. However, up to now, this field has never been used to perform optical nonlinearities, the so-called "evanescent nonlinearities", except in two very recent communications $[18,19]$. In these studies we focused on the Raman effect as the "evanescent nonlinearity": the evanescent field of the guided mode excited the Raman effect in a liquid (ethanol or toluene were chosen), in which the nanofibre was immersed. This Raman effect indeed presents the main advantage of being automatically phase-matched. Implementing an experiment to evidence this Raman effect is therefore relatively easy, as we do not need to engineer the dispersion properties of the nanofibre.

The purpose of the present paper is to design such an experiment. In Section 2, we first detail the scheme and the main features of the optical arrangement suggested to evidence the Raman evanescent nonlinearity. In Section 3 we model the Raman interaction in the evanescent field. It allows us to determine the parameters of the nanofibre that are required for efficient generation of the nonlinear effect: its diameter, its uniformity and its length. Examples of expected Raman gains along with the typical parameters required to observe this effect will then be given in Section 4. Based on the knowledge of these Raman gains, Section 5 details the evolution of optical pulses as they propagate along the nanofibre. 


\section{SCHEME OF A RAMAN EVANESCENT NONLINEARITY}

A typical scheme for exploring the Raman evanescent nonlinearity with a tapered optical fibre is depicted in Figure 1.

The light propagates from left to right. The pump beam first propagates as the fundamental $H E_{11}$ core mode of the unstretched fibre. Most of the energy is confined in this core whose diameter is typically $9 \mu \mathrm{m}$, while the cladding diameter is around $125 \mu \mathrm{m}$ for a telecom fibre. The light then enters the left taper section. This reduction of the fibre dimensions along this taper modifies the mode transverse structure [20]. When the fibre reaches a diameter smaller than or about the wavelength, a significant part of the mode propagates as an evanescent field, that is to say propagates outside the silica, in the surrounding medium. The mode then reaches the nanofibre waist that can either be just localized at the junction between the two tapers or may extend over a few centimetres. For these small diameters, the nanofibre can be assimilated to a two-layer fibre consisting in a glass core, originating from the initial fibre cladding, and a new cladding made of the Raman medium in which the nanofibre is immersed. The original core can indeed be totally neglected after stretching, its diameter being much lower than the wavelength. Because of the small radius of the core in this section, the nanofibre is single mode: the $H E_{11}$ mode of the un-stretched fibre has been transformed into the $H E_{11}$ mode of the stretched nanofibre. Although being denominated by the same name, $H E_{11}$, their shapes differ. In the stretched section this mode possesses a strong evanescent field that feels the external Raman medium and activates the Stimulated Raman Scattering. Spontaneous Raman Scattering initiates this process: by interacting with the molecular vibrations, the incident pump photons are scattered under the form of other photons of lower energy. Some of these scattered photons are also guided by the nanofibre. They are then amplified at the expense of the initial pump beam through the stimulated Raman amplification [21]. By this process, one may expect to convert a large part of the initial pump beam into a Stokes beam of a longer wavelength. Both beams are then collected by the right taper, propagate in the other un-stretched part of the fibre as core modes of this un-tapered fibre section and can be subsequently analysed.

What is the transverse structure of the excited Stokes wave? The problem is as follows. The nanofibre is single mode. That means that it possesses only one bounded mode, the fundamental $H E_{11}$ transverse mode $[22,23]$. However besides this mode, radiation and leaky modes also exist. In Figure 2, we plotted the $H E_{11}$ pump and Stokes intensities for modes polarized along the transverse $x$ axis. The nanofibre is immersed in ethanol and its radius is equal to $\rho_{0}=200 \mathrm{~nm}$. The $x$ axis in Figure 2 is expressed in radius units, i.e. normalized to $\rho_{0}$, so that a radius of unity corresponds to the nanofibre boundary. The Raman susceptibility excited by the pump beam in the surrounding medium is represented as a shaded area. This figure underlines the imperfect overlap between the excitation and the fundamental $H E_{11}$ Stokes mode. This imperfect overlap implies that non-zero overlaps with higher Stokes transverse modes exist and should be taken into account. Indeed although the single-mode nanofibre sup-

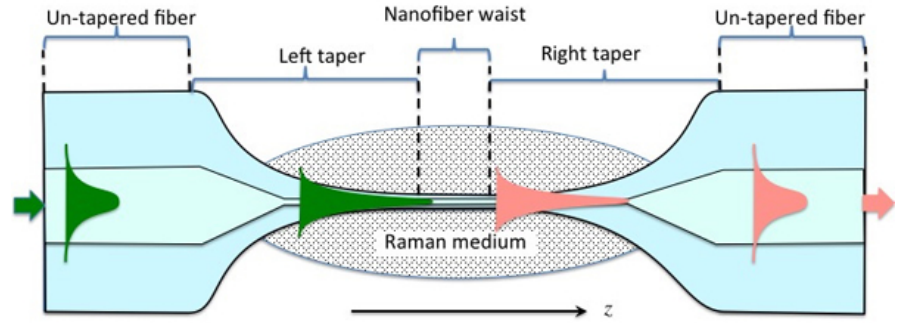

FIG. 1 Typical scheme to evidence the Raman evanescent nonlinearity with a tapered nanofibre.

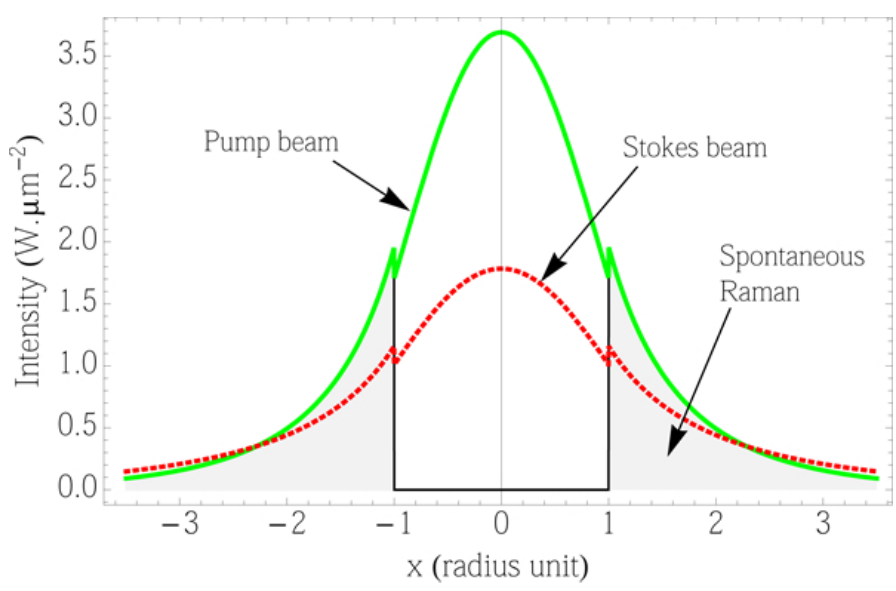

FIG. 2 Illustration of the imperfect overlap of the intensity of the $\mathrm{HE}_{11}$ mode at the Stokes wavelength (dotted red line) with the Raman spontaneous scattering medium (shaded area) excited by the $\mathrm{HE}_{11}$ mode at the pump wavelength (green full line). The pump and Stokes powers are both assumed to be equal to $1 \mathrm{~W}$.

ports only one bounded mode, the fundamental $H E_{11}$ mode, other non-bounded modes also exist and should be considered [23].

We may therefore anticipate the energy coupling from the pump $H E_{11}$ mode to a multiplicity of transverse Stokes modes. However, we have to consider the losses and the gains experienced by these higher modes. Typically, we will demonstrate that the product "Raman gain $x$ power" is smaller or about $g_{R} P_{P} \approx 100 \mathrm{~m}^{-1}$ while the attenuation coefficient is larger by at least two orders of magnitude for any mode except the fundamental mode for which the attenuation is negligible over propagation lengths of a few centimetres, as considered here. Because their attenuation is much larger that the Raman gain, the higher modes disappear before taking away any energy from the pump. We conclude that, even if the modal Raman gain is large for a non-bounded mode, it does not contribute to the pump depletion because the "gain minus loss" parameter is negative. We can thus neglect all these non-bounded modes in our analyses. Two important consequences of this conclusion are:

- The higher order modes do not contribute and thus do not take away any energy;

- The Stokes wave presents the shape of the fundamental $H E_{11}$ mode of the single mode nanofibre, whatever the exact shape of the excited region. 


\section{MODELLING}

\subsection{Assumptions}

\subsubsection{Raman effect and other competing nonlinear effects}

Besides the expected evanescent Raman effect, other nonlinear effects may occur within the scheme depicted in the above Figure 1. One of these effects, which could eventually prevent the observation of a Raman evanescent effect, may originate from the Kerr optical nonlinearity, as is the case for parametric wave-mixings and instabilities, phenomena at the origin of supercontinuum generation [21]. These wave-mixing processes are phase-sensitive: a phase-matching condition must be fulfilled to efficiently excite them. This phase matching condition has been deeply investigated previously. In case of a silica nanofibre with air as the external medium, a zero dispersion wavelength of $532 \mathrm{~nm}$ is obtained for a nanofibre radius of about $500 \mathrm{~nm}$ [4]. We calculated that immersing such a nanofibre in ethanol, for example, decreases the dispersion down to about $D \approx-1000 \mathrm{ps} . \mathrm{nm}^{-1} \cdot \mathrm{km}^{-1}$, value for which parametric instabilities are unlikely to happen.

Similarly, the silica Raman scattering could also be excited. Here, we anticipate our results that most often demonstrate that the evanescent field Raman effect appears in the surrounding material for a lower power than for the Raman excitation of the silica core. This is due to the low Raman coefficient of silica.

From now on, we can neglect these other nonlinear effects and focus our discussion on the Raman effect in the surrounding medium.

\subsubsection{Backscattering Raman effect}

Backscattered Stimulated Raman Scattering is not forbidden and could eventually be observed. However we never saw it in our previous experiment. The reason is probably that, given that the nanofibre length is not negligible compared to the pump pulse length, the temporal overlap between the pump and a forward Stokes beam is larger than between the pump and a backscattered beam. In our experiments, the threshold for the onset of the backscattered SRS is thus higher than for the forward SRS. Typically, the pulse lengths were about $10 \mathrm{~cm}$ long while the nanofibres were also about $10 \mathrm{~cm}$. Neglecting the backscattered waves simplifies the simulations and still correctly reproduces our experimental conditions.

\subsubsection{Pump beam characteristics}

Light propagation inside tapers has already been investigated in depth [23]. For adequately designed tapers, called adiabatic tapers, the modes are progressively transformed with negligible loss from the un-stretched fibre core mode to the nanofibre mode. Typically transmissions larger than $90 \%$ for the whole device, including the two tapers and eventually a nanofibre waist a few centimetres in length, are not unusual. Because of this adiabaticity we exactly control the mode injected in the nanofibre by controlling the injected light in the left taper. We can, in particular, ensure that the injected pump $H E_{11}$ mode is not polarization degenerated. Given that for any liquid the scattered Raman component along the pump polarization is always larger than along the orthogonal polarized scattered component, we model light propagation taking into account that both the pump and the Stokes waves are $H E_{11}$ modes sharing the same polarization.

The pump wavelength is set at $532 \mathrm{~nm}$ as this wavelength is largely available and has been used for our first experimental demonstration [19]. With this pump wavelength, the Stokes wavelength also lies in the visible range. This visible region allows using many Raman liquids (such as water, ethanol, methanol, benzene etc.) whose transparency is good in the visible. This would not be the case if a $1064 \mathrm{~nm}$ laser were used. However transposing our results to other wavelengths is straightforward.

We also assume that the pump pulse width is much longer than the relaxation time of the Raman effect. This was largely verified in the first demonstration as the pump pulse line-width was lower than $1 \mathrm{GHz}$, and the Raman natural linewidth of the main line of ethanol was about $522 \mathrm{GHz}$. In the following simulations, the pulse shape is assumed to be an hyperbolic secant whose full width at half maximum is 500 ps. The experiments were thus conducted in the quasi-continuous regime allowing us to describe them with a coupled-wave approach, as detailed below.

\subsection{Coupled-wave analysis}

\subsubsection{Coupled-mode equations versus Schrödinger equation}

The nonlinear effects in optical fibres are quite often treated starting from the nonlinear Schrödinger equation, NLS equation, describing the evolution of the total optical electric field during its propagation through the fibre [21]. The resolution of this NLS equation very conveniently describes the creation of supercontinuums in which an initial pump beam breaks over a very large spectrum. However, if only a few discrete frequencies are present, the couple-mode approach is more convenient. This is the case for the problem we investigate in which the initial pump beam with a very "narrow" spectrum is transformed into a single or a few Stokes beams, each of them with a "narrow" spectrum. By very "narrow" spectrum we mean that the spectrum of the pump beam is much narrower than the Raman linewidth. We thus consider that, over these narrow spectra, the Raman gain and the refractive indices are the ones of the central wavelength of each bandwidth. These considerations allow us to follow a simpler coupled-mode approach.

\subsubsection{Coupled-mode equations}

Working far away from the absorption bands of the Raman medium, we neglect all the attenuation factors. Although the Raman spectrum of any material presents a complex structure made of several lines and bands, we only take into account its main Raman line. Indeed, when the pump is not yet depleted by the Raman effect, the Stokes powers evolve exponentially. Therefore, only a moderate difference between the gains of two Raman lines produces a very large difference in 
the output powers of these two lines. Most often, the main Raman line predominates and supersedes any other one.

It is worth remembering that a nanofibre is indefinitely single mode. Nothing prevents the apparition of a Raman cascade: by interacting with the medium, the pump beam can produce a first Stokes order, at angular frequency $\omega_{S 1}$, this first Stokes order can itself be Raman shifted to produce the second Stokes order at $\omega_{S 2}$ and so on. If $\omega_{P}$ is the pump angular frequency, $\Delta \omega_{\text {Stokes }}$ the Stokes angular frequency shift, we have:

$$
\begin{gathered}
\omega_{S 1}=\omega_{P}-\Delta \omega_{\text {Stokes }}, \quad \omega_{S 2}=\omega_{S 1}-\Delta \omega_{\text {Stokes }}, \\
\omega_{S 3}=\omega_{S 2}-\Delta \omega_{\text {Stokes }}, \cdots
\end{gathered}
$$

Taking into account the two first Stokes orders, we can express the set of coupled-wave equations in the quasi-cw regime. With $z$ being the coordinate along the nanofibre, these equations are:

$$
\left\{\begin{array}{l}
\frac{\mathrm{d} P_{P}}{\mathrm{~d} z}=-\frac{\omega_{P}}{\omega_{S 1}} g_{S 1} P_{P} P_{S 1} \\
\frac{\mathrm{d} P_{S 1}}{\mathrm{~d} z}=g_{S 1} P_{P} P_{S 1}-\frac{\omega_{S 1}}{\omega_{S 2}} g_{S 2} P_{S 1} P_{S 2} \\
\frac{\mathrm{d} P_{S 2}}{\mathrm{~d} z}=g_{S 2} P_{S 1} P_{S 2}
\end{array}\right.
$$

$P_{P}, P_{S 1, S 2}$ are the instantaneous powers of the pump and of the two Stokes waves. To express the modal Raman gains, $g_{S 1, S 2}$, we follow the analysis conducted in [24] that takes into account the vectorial nature of the optical electric fields. In this analysis, the modal Raman gains between the pump and the first Stokes order, and between the first and second Stokes orders, are expressed by:

$$
\left\{\begin{array}{l}
g_{S 1}=\frac{\varepsilon_{0} c^{2} \iint_{\text {active area }} g_{R, S 1} n^{2}\left|\mathbf{e}_{P} \cdot \mathbf{e}_{S 1}\right|^{2} \mathrm{~d} A}{\iint_{\text {total area }}\left(\mathbf{e}_{P} \times \mathbf{h}_{P}\right) \cdot \hat{\mathbf{z}} \mathrm{d} A \iint_{\text {total area }}\left(\mathbf{e}_{S 1} \times \mathbf{h}_{S 1}\right) \cdot \hat{\mathbf{z}} \mathrm{d} A} \\
\varepsilon_{S 2}=\frac{\varepsilon_{0} c^{2} \iiint_{\text {activearea }} g_{R, S 2} n^{2}\left|\mathbf{e}_{S 1} \cdot \mathbf{e}_{S 2}\right|^{2} \mathrm{~d} A}{\iint_{\text {total area }}\left(\mathbf{e}_{S 1} \times \mathbf{h}_{S 1}\right) \cdot \hat{\mathbf{z}} \mathrm{d} A \iint_{\text {total area }}\left(\mathbf{e}_{S 2} \times \mathbf{h}_{S 2}\right) \cdot \hat{\mathbf{z}} \mathrm{d} A}
\end{array}\right.
$$

in which, $\varepsilon_{0}$ and $c$ are the dielectric permittivity and the light celerity in vacuum; $\mathbf{e}_{P}, \mathbf{h}_{P}, \mathbf{e}_{S m}$ and $\mathbf{h}_{S m}$ are the vectorial electric and magnetic fields of the pump and of the $m^{\text {th }}$ Stokes order. For the two-layer structure we discussed here, their expressions are listed in the literature $[22,23]$.

$g_{R, S m}$ are the Raman coefficients at the peak of the Raman line. They depend on the Stokes wavelength. We have derived their values for a series of liquids and mixtures in our Appendix. Knowing these values for a given Stokes wavelength and for a given fibre radius, we can compute the structure of the fundamental modes for the pump and Stokes beams and then derive the modal Raman gain from Eq. (3).

The Raman coefficients $g_{R, S m}$ are expressed in $\mathrm{m} \cdot \mathrm{W}^{-1}$, while the modal Raman gains $g_{S m}$ are expressed in $\mathrm{m}^{-1} \cdot \mathrm{W}^{-1}$. As the nanofibre radius varies along with the $z$ axis, the modal Raman gains are function of $z$.

The integrals in Eq. (3) are calculated over the transverse cross-section of the beams. The integration of the numerator of Eq. (3) is conducted only over the Raman active area, while the integrations of the normalization factors in the denominator are conducted over the full transverse plane.

\subsubsection{Simulations of the spontaneous scattering}

The stimulated spectrum results from the amplification of the spontaneous Raman scattering along the fibre. This spontaneous scattering can be calculated from the knowledge of the Raman cross-section [25]. It can then be introduced in the right-hand side of the set of Eq. (2) by replacing $P_{S n}$ with $P_{S n}+R_{S n}$. As explained in [25], the expression of the scattering terms $R_{S n}$ is a function of the Raman gain, of the Stokes linewidth and of the fibre parameters. An alternative to this procedure was proposed in [26, 27]. Reference [26] demonstrates that the net result of the spontaneous emission is equivalent to the introduction of a seed term, $P_{S n}(z=0)$ for the Stokes wave powers at the fibre input. The two approaches have been demonstrated to lead to the same result, that is to say the same evolution of the pump and Stokes intensities along the fibre. We extend the value for the seed term derived in [25] to the case of a modal Raman gain varying along the fibre axis. If $L$ is the nanofibre length, with $\hbar$ the reduced Plank constant, this seed term for the first Stokes is expressed by [27]:

$$
P_{S 1}(z=0)=\frac{\sqrt{\pi} \hbar \omega_{S 1} \Delta v_{F W H M}}{2 \sqrt{\int_{0}^{L} g_{S 1} P_{P}(z=0) \mathrm{d} z}},
$$

with $\Delta v_{F W H M}$ the full width at half maximum of the Raman line.

We do not have such a formula for the second Stokes order. Indeed, this second Stokes is excited by the first one, whose power is zero at the fibre input. Nevertheless, once the Raman onset is exceeded, the first Stokes power becomes comparable to the input pump power. For the second Stokes, we thus use a seed term, $P_{S 2}(z=0)$, calculated from Eq. (4), taking into account the angular frequency and Raman gain for the second Stokes order, but keeping the value of the pump power $P_{P}(z=0)$. This second seed term is thus approximate but the evolution of the Stokes powers versus the pump power is not very sensitive to the accuracy of this seed value. We indeed checked that even a change of this term by a factor of 10 does not significantly change the results presented below.

\section{MODAL RAMAN GAIN}

\subsection{General parameters}

As discussed above, when the fibre is tapered down, its original core diameter becomes much smaller than the wavelength. Therefore, the exact type of fibre, i.e. its core structure, is of negligible importance. Only the cladding glass matters. In our previous demonstration, we used a SMF-28 telecom fibre. To stick to this previous demonstration, we thus consider that the nanofibre is made of fused silica. For the ease of the demonstration, the surrounding Raman medium is chosen to be liquid.

In order to guide light in such a glass nanofibre the refractive index of this liquid must remain smaller than that of silica, whose refractive index at $532 \mathrm{~nm}$ is 1.461 . This is possible in a few pure liquids, such as water, methanol, ethanol, acetone... We first examine this case of pure liquids in the following section. 
Using high refractive index liquids is also of interest because it allows reaching new Stokes wavelengths. This is for instance the case of benzene derivatives whose refractive indices are larger than that of silica. To perform the evanescent Raman conversion one solution consists in mixing this liquid with a solvent of lower refractive index so that the refractive index of the mixture remains lower than the one of the fused silica. This is the second case we examine in the following section.

Although the Raman effect has been widely investigated, the Raman coefficients of liquids are not necessarily known in the wavelength range we are interested in. We computed some of them from measurements published in the literature at other wavelengths and in other configurations. The derivation of the relevant equations, of the coefficient values and of the pertaining references are reported in the Appendix.

\subsection{Modal Raman gain versus the refractive index of the surrounding liquid}

We first consider two pure liquids, ethanol and methanol. The Stokes wavelengths for ethanol and methanol are nearly the same. These two liquids mainly differ by their refractive indices. To derive the modal Raman gains of a nanofibre immersed in these liquids, we start determining the shapes of the fundamental pump and of the Stokes $H E_{11}$ modes for the first and second order. Then, using Eq. (3), we compute the modal Raman gains, $g_{S 1}$ and $g_{S 2}$, on the first main Stokes line. In Figure 3, we plotted these modal Raman gains versus the nanofibre radius. Red and blue lines are respectively for ethanol and methanol. The Raman gains for the first Stokes orders are represented in full lines, and the dashed lines are for the second Stokes orders.

For each liquid and each order there is an optimum radius. For radii larger than this optimum, the modal gain decreases because the evanescent field is reduced, the mode being more confined in the glass core. For radii smaller than the optimum, the evanescent field spreads over larger distances so that its amplitude diminishes: the modal gain decreases once again. For the first order, one sees that, although the Raman coefficient of methanol is smaller than that of ethanol, the maximum of the modal Raman gain is about the same for methanol and for ethanol. This effect is even more prononced on the second Stokes order for which the modal Raman gain peaks at a higher value for methanol. The reason lies in the larger refractive index of ethanol that makes the mode spread over a larger area. Nevertheless, for large radii, the modal gain with ethanol becomes larger than for methanol for the same reason: the lower refractive index of methanol makes the mode more confined in the core and thus decreases the gain.

A quite interesting consequence of this feature is that the ratio $g_{S 1} / g_{S 2}$ strongly depends on the fibre radius. One could for instance select a radius to get a large ratio. This will increase the energy transfer between the pump and the first Stokes order before this Stokes order is depleted by the second order.

Let us now examine the evolution of the Raman gain on the first Raman order in case of a mixture of liquids. We first illustrate this case with the Raman excitation on the first line of

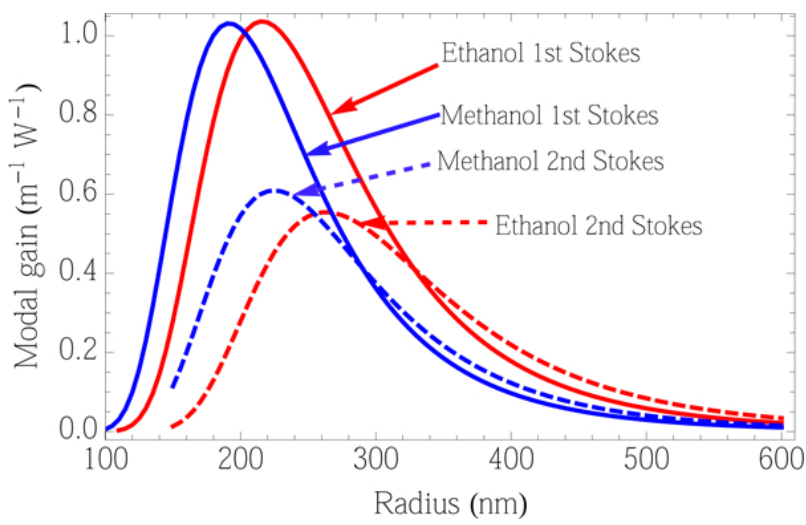

FIG. 3 Modal Raman gain experienced by the guided $\mathrm{HE}_{11}$ mode versus nanofibre radius for immersion in ethanol and methanol for the first and second Stokes orders.

toluene in a mixture of toluene and ethanol. Toluene is thus the active Raman medium and ethanol is just used to lower the refractive index of the mixture below the one of silica. Computing the values of the Raman coefficient of toluene in the mixture is not totally straightforward. Indeed, the Raman coefficient of toluene in the mixture is proportional to the fraction volume of toluene, and to the Raman cross-section of the toluene molecule in the mixture. Because the local field experienced by a molecule depends on its environment, this crosssection is a nonlinear function of the refractive index of the mixture. We derived the Raman coefficient of a compound in a mixture in our Appendix, Eq. (A5), taking into account the refractive index of the mixture with Eq. (A8). Once we know this Raman coefficient we proceed as previously. We first determine the shapes of the fundamental pump and Stokes $H E_{11}$ modes, then, using Eq. (3) we compute the modal Raman gain of the compound in the mixture.

In Figure 4(a), we thus plotted the modal Raman gain of a nanofibre in a mixture of toluene and ethanol versus the volume fraction of toluene and the nanofibre radius. Any vertical profile of this map, i.e. at a given value of the volume fraction of toluene is a bell shaped curve, similar to the curve discussed previously and shown in Figure 3. A cut at a given radius also presents a bell shape. For a volume fraction of toluene lower than the optimum, the decay of the modal Raman gain results from the decay of the Raman coefficient of toluene in the mixture. For a larger volume fraction, the modal Raman gain also decays because of the increase of the refractive index of the mixture. This increase makes the mode spread over a larger area in the liquid. The numerator of Eq. (3) also decreases and so does the modal Raman gain. Above a volume fraction of about 0.72 , the refractive index of the mixture equals the refractive index of fused-silica for the Stokes wavelength: the mode is no more guided. Below a fraction volume of $8 \%$, the modal Raman gain of ethanol in the mixture predominates.

In order to understand the importance of the refractive index of the solvent in the mixture we also plotted the Raman modal gain in case of a mixture of toluene in methanol, instead of ethanol, see Figure 4(b). Clearly, compared to methanol, the higher refractive index of ethanol is detrimental. Even if the refractive index of ethanol is larger by only 0.02 , the maxi- 

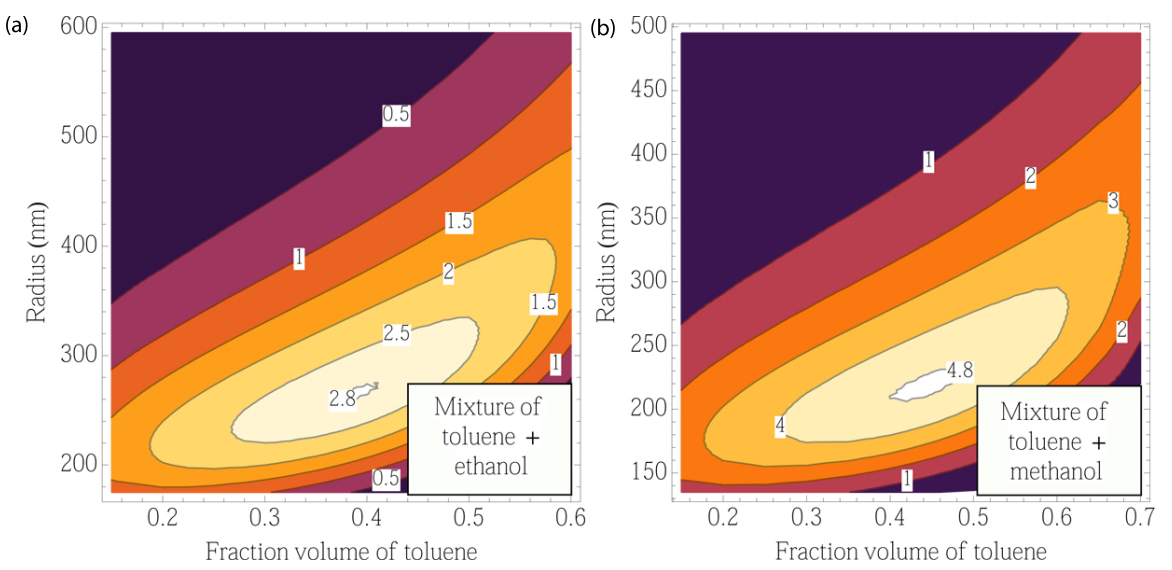

FIG. 4 Modal Raman gain $\left(\mathrm{m}^{-1} \cdot \mathrm{W}^{-1}\right)$ of a nanofibre on the main Raman line of toluene diluted in ethanol (a) or methanol (b).
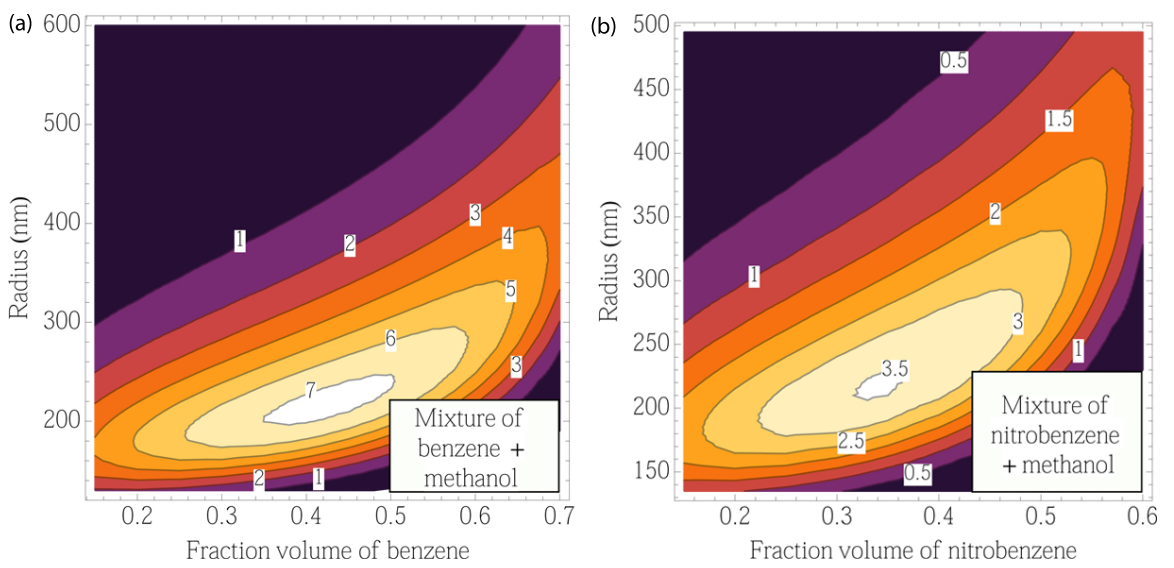

FIG. 5 Modal Raman gain $\left(\mathrm{m}^{-1} \cdot \mathrm{W}^{-1}\right)$ of a nanofibre on the main Raman line of benzene diluted in methanol (a) and nitrobenzene diluted in methanol (b).

mum Raman gain is reduced by nearly a factor 2! Indeed a lower refractive index of the solvent allows the use of a higher volume fraction of toluene resulting in higher gains.

Correctly selecting a solvent with a refractive index as low as possible is thus of first importance to maximize the modal Raman gain.

We also plotted the modal Raman gain of a nanofibre immersed in benzene, or nitrobenzene, dissolved in methanol, see Figure 5. The Raman coefficient of benzene is larger than the one of toluene by a factor 1.6 while the refractive index and Stokes wavelengths are about the same for the two liquids (see appendix). Consequently, as can be observed in Figure 5(a), its maximum modal Raman gain, 7.3, is about 1.5 times larger than for toluene and is located at the same position. The Raman coefficient of nitrobenzene is lower than the one of benzene, and its refractive index is higher. Compared to benzene, this large refractive index further lowers the maximum modal gain and shifts it to a lower fraction volume, see Figure 5(b).

\section{SIMULATIONS OF THE PULSE EVOLUTION ALONG THE NANOFIBRE}

\subsection{Conditions of simulations}

The objective of this paper is to investigate the feasibility of the evanescent Raman interaction with nanofibres and to determine what are the required nanofibre characteristics to achieve such a demonstration. While detailing the evanescent Raman interaction and the scheme depicted in Figure 1, we have considered that the evanescent interaction may take place both in the taper region and in the nanofibre waist section. This is the reason why the modal gain appearing in the coupled-wave Eq. (2) has an implicit $z$ dependence. In the following, in order to derive simple expressions, we assume that the main Raman contribution arises from the uniform nanofibre waist only, so that the modal Raman gain is constant along the nanofibre. Extension to other nanofibre shapes, including the Raman contribution from the tapers will be straightforward from Eq. (3).

\subsection{Continuous versus pulsed experiments} We study stimulated Raman scattering so that no Stokes waves are injected at the nanofibre input $z=0$. For these Stokes waves, the only terms to take into account at this boundary are the seed terms expressed by Eq. (4). These terms depend on the input pump power.

As an example, we consider a uniform nanofibre immersed in ethanol whose length is $L=10 \mathrm{~cm}$ and radius is $\rho=215 \mathrm{~nm}$. The modal Raman gain is at its maximum and is equal to $g_{S 1} \approx 1.04 \mathrm{~m}^{-1} \cdot W^{-1}$ for the first Stokes. It is equal to $g_{S 2} \approx 0.391 \mathrm{~m}^{-1} . \mathrm{W}^{-1}$ for the second Stokes. 

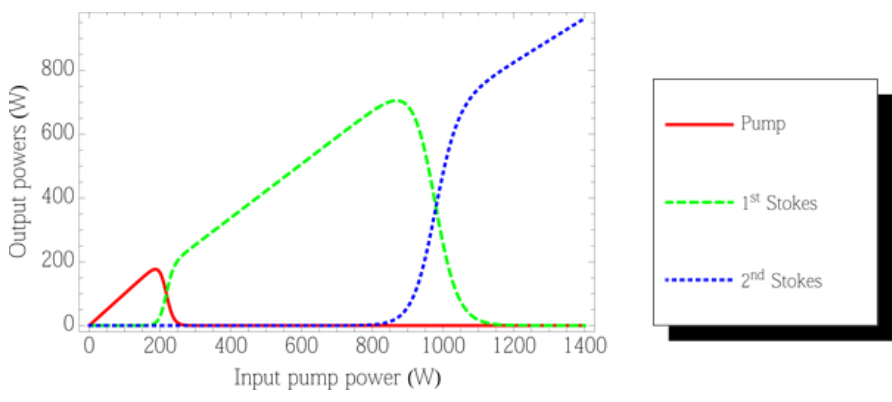

FIC. 6 Evolution of the powers in a cw experiment for a nanofibre immersed in ethanol whose length is $10 \mathrm{~cm}$ and radius is $215 \mathrm{~nm}$.

\subsubsection{Continuous excitation}

We first solve the coupled-wave equations assuming a cw beam. Although working with cw beams is probably unrealistic because of the high average powers required, these results will help us to analyse our next predictions with pulses. In Figure 6, we plotted the transmitted pump, $1^{\text {st }}$ Stokes and $2^{\text {nd }}$ Stokes powers versus input pump power.

In the literature, the Raman critical power is reported as being the input power for which the output pump power equals the first Stokes power [26]. According to this definition the critical power for the first Stokes as found on the curves in Figure 5 is $P_{\text {crit }} \approx 220 \mathrm{~W}$, corresponding to a critical product $\gamma=g_{S 1} P_{\text {crit }} L$ of:

$$
\gamma=g_{S 1} P_{\text {crit }} L \approx 23
$$

This last value for the critical power in the cw regime is in total agreement with the value found with the analytical formula that is obtained by solving the following equation [26]:

$$
\gamma^{3 / 2} e^{-\gamma}=\frac{\sqrt{\pi}}{2} h v_{S} g_{S 1} \Delta v_{F W H M} L
$$

$v_{S}$ being the frequency of the Stokes wave and $h$ the Planck's constant.

It is interesting to note that this value for $\gamma$ is much larger than the values usually reported in Raman experiments in fibres, about $\gamma \approx 16$ [21]. This difference originates from the larger gains and smaller lengths than those commonly used, as can be deduced from analysing Eq. (6).

\subsubsection{Pulsed excitation}

Because the pump pulse width is much longer than the relaxation processes and because we deal with nanofibres whose lengths are smaller than the dispersion length, we can follow [27] and represent these long pulses by discretizing them on a discrete grid of temporal points: we treat each value of the power along the pulse shape in a cw regime as done in the previous section. Therefore, the curves plotted for the cw beams in Figure 6 are also valid for any input power along this temporal grid. Following this procedure we plotted in Figure 7 the temporal shape of the transmitted pump and Stokes pulses for a pump pulse peak power of $1000 \mathrm{~W}$ (corresponding to a total input energy of $0.56 \mu \mathrm{J})$.

The horizontal dashed line stands for the critical power, $P_{\text {crit }} \approx 220 \mathrm{~W}$. One sees that when the pump power is be-

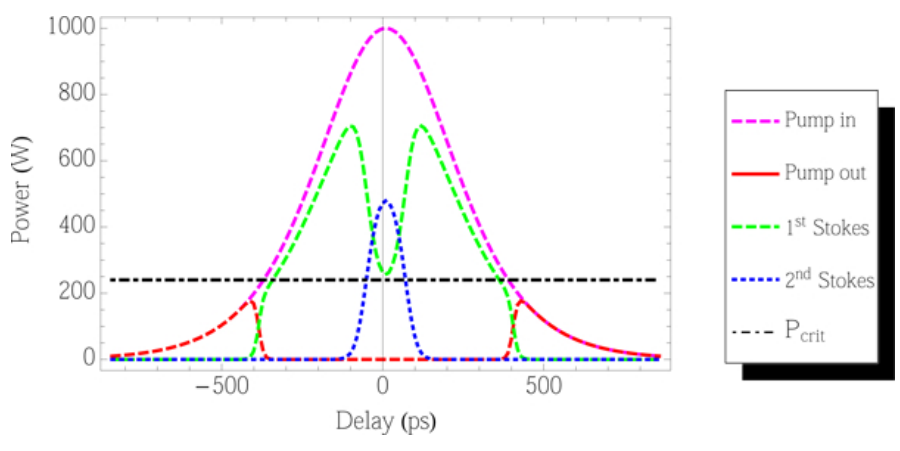

FIG. 7 Examples of the temporal shapes of the various involved pulses (see insert) in a nanofibre immersed in ethanol whose length is $10 \mathrm{~cm}$ and radius is $215 \mathrm{~nm}$.

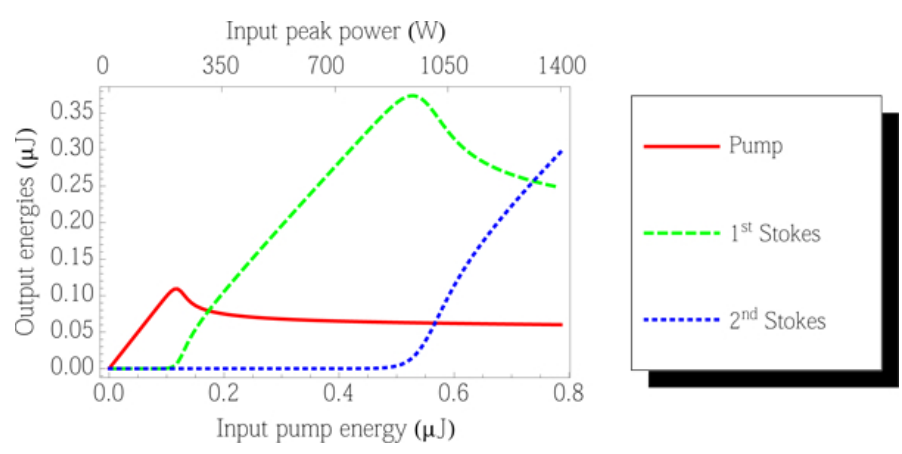

FIG. 8 Evolution of the pulse energies versus the input pump energy and peak power, in a nanofibre immersed in ethanol whose length is $10 \mathrm{~cm}$ and radius is $215 \mathrm{~nm}$.

low this critical power, on the aisles, the pump pulse remains largely un-depleted, while it is nearly depleted around the middle of the pump pulse.

The pulse energy is subsequently calculated by integrating the pulse power over time. We can thus plot the evolution of the pulse energies versus the input pump energies (bottom axis in Figure 8) and, equivalently versus the input pump peak power (top axis in Figure 8).

One sees that the Stokes starts to significantly depart from zero for a peak power of about $200 \mathrm{~W}$, which is consistent with Figure 6. Around this point, only the intensity around the peak of the pump beam significantly contributes to the Stokes waves.

Similarly to the critical power defined for cw waves, one can define a critical energy as being the input pump energy for which the output pump and Stokes waves are the same. When increasing the pulse pump energy, the critical power is reached at the peak before the aisles. That is why the critical energy in the pulse regime is reached for an input peak power of about $320 \mathrm{~W}$ that is larger than in the $\mathrm{cw}$ regime.

For the same reason, energy is always left in the pump aisles even at high power so that the pump beam can never be completely depleted conversely to the behaviour observed in the cw regime. 


\section{DISCUSSION AND CONCLUSION}

We can now easily design an experiment using nanofibres to excite a Raman evanescent nonlinearity. It is worth noting that, in spite of the fact that the Raman scattering occurs in the surrounding medium only, the excited Stokes wave is a fundamental mode, with large intensity in the core. We performed our simulations with ethanol. Its Raman coefficient is relatively low but it is readily available, easy to handle liquid. Despite this low coefficient, the required critical peak power is relatively moderate, a few hundred watts for a $10 \mathrm{~cm}$ long nanofibre. These powers are easily available even with lowcost Q-switched sub-nanosecond microlasers. Furthermore, even with 500 ps pulses, the average energy per pulse remains lower than the damage threshold of silica as evidenced by preliminary experiments [19]. Indeed although this subnanosecond regime is known to be worse than the picosecond or even the femtosecond regime with regard to the silica laser damage, the Raman threshold is reached before damaging the nanofibre [19].

The required diameters, a few hundred nanometres, are within reach of optimized pulling platforms even over several centimetres. It is worth stressing that this diameter does not require to be set with a great accuracy: as illustrated in Figures 3 and 4, a change of the diameter by about $\pm 10 \%$ around the optimum value changes the modal Raman gain by less than $\pm 10 \%$.

Stimulated Raman scattering can also be excited in a liquid filling the core of hollow core microstructured fibres [28]-[30]. The critical product $\gamma$ is about the same as for a nanofibre. Nevertheless, because the modal Raman gain of microstructured fibres is about ten times lower, a length ten times larger is required to observe the same effect with the same input power. These larger Raman gains in nanofibres result from a stronger light focusing and consequently a larger sensitivity to laser damage thresholds. Working with large powers is thus probably easier with microstructured fibres. Furthermore, nanofibres do not present the band structure, which in microstructured bandgap fibres allows to stop the Raman cascade and accumulate all the Stokes power on a single line [29].

Nevertheless, nanofibres should be considered as an alternative to hollow core microstructured fibres. We foresee many advantages of using nanofibres among which:

- Tapering the same nanofibre in successive sections allows cascading several evanescent non-linear effects;

- The surrounding liquid can be readily changed;

- Larger nanofibres could even be used to increase the resistance to the laser silica damage, for instance by using $E H_{11}$ or even a TM mode that still present an evanescent field for larger nanofibre diameters;

- The fabrication process is considerably simpler for nanofibres than for holey fibres, allowing more flexibility in the choice of the fibre material.

Given the large choice of available materials for the medium surrounding the nanofibres, as well as for nanofibre materials, these considerations open the way to the exploration of a new class of experiments and devices using these evanescent nonlinearities, including Raman nonlinearities and parametric nonlinearities.

\section{APPENDIX}

\subsection{Introduction}

In this appendix, we detail the derivation of the Raman coefficients of pure liquids and of mixtures of liquids. These Raman coefficients depend on the optical configuration: polarizations and angle between the pump and the Stokes waves.

In the first section, we give the expression of the Raman coefficient in our optical configuration: forward Raman scattering with the Stokes wave being polarized along the pump beam linear polarization. This expression can be computed from measurements found in the literature. In the next section, we collect some references in which these measurements can be found for a small set of liquids. We then summarize the results of the computation giving the Raman coefficients in a short table. To finish with, in the last section, we derive the formulae required to compute the Raman coefficient of a mixture as a function of the Raman coefficients of the mixture compounds and of their refractive indices.

\subsection{Expression for the Raman gain Coefficient}

The Raman coefficient depends on the linewidth of the pump beam. In all values given hereafter, we assume this value to be much smaller than the Stokes linewidth. Quite often, the values reported in the literature have not been obtained at the wavelength of interest, $532 \mathrm{~nm}$ in the example given in this paper. We thus have to compute them from the data obtained at another wavelength and from theoretical formulae. According to [31], the theoretical expression for the Raman coefficient is:

$$
g_{S}=\frac{8 \pi}{\Delta v_{F W H M}} \frac{N c^{2}}{\omega_{S}^{3} \hbar n_{S}^{2}}\left(\frac{\partial \sigma}{\partial \Omega}\right)_{T D S C}^{F}
$$

with $c$ the velocity of light, $\omega_{S}$ and $n_{S}$ the Stokes angular frequency and refractive index, $N$ the number of molecules per unit volume.

The last term in Eq. (A1) is the forward Total Differential Scattering Cross-section, TDSC, which is a value integrated over the full Raman line and experienced by the Stokes beam that co-propagates with the pump beam.

We need to consider the polarization of the pump and Stokes waves. Our pump beam propagates under a fundamental $H E_{11}$ mode. One can always decompose the Stokes beam on the two orthogonally polarized fundamental $H E_{11}$ modes. We note them $H E_{11}^{/ /}$and $H E_{11}^{\perp}$ if they are polarized along the pump polarization or perpendicular to it. The TDSC for these two modes are different. We note them $(\partial \sigma / \partial \Omega)_{T D S C}^{F, / /}$ and $(\partial \sigma / \partial \Omega)_{T D S C}^{F, \perp}$. For most liquids and Raman lines, none 
of these two coefficients is zero so that the spontaneous Raman emission is coupled to the two $H E_{11}^{/ /}$and $H E_{11}^{\perp}$ modes. However, the $(\partial \sigma / \partial \Omega)_{T D S C}^{F, / /}$ is always the largest so that the stimulated Raman scattering, which is an exponential process, occurs on $H E_{11}^{/ /}$mainly. The polarization of the Stokes beam is thus the same as the pump beam polarization. This is the reason why we only took into account the "parallel" Raman scattering in Eq.(3).

How should we compute this "parallel" Raman coefficient from the values published in the literature?

One concern is that the TDSC scattering indicatrix is not spherical [32]. Its value depends on the direction of observation relative to the direction of the pump beam. We are thus interested in the forward TDSC, $(\partial \sigma / \partial \Omega)_{T D S C}^{F}$, while most often, the tabulated values correspond to the light scattered at $90^{\circ},(\partial \sigma / \partial \Omega)_{\text {TDSC }}^{90^{\circ}}$

We thus have to derive the expression we required, $(\partial \sigma / \partial \Omega)_{T D S C^{\prime}}^{F, / /}$ from the measured values, $(\partial \sigma / \partial \Omega)_{T D S C^{\prime}}^{90^{\circ}}$ found in the literature. This $90^{\circ}$ TDSC takes into account both polarizations. An interesting feature is that, quite often, the $90^{\circ} \mathrm{TDSC}$ is measured in the $90^{\circ}$ direction perpendicular to the pump beam polarization. In such a case, the found value for the TDSC is isotropic (see ref [32]). It does not depend on the observation direction. It is thus equal to its forward value:

$$
\left(\frac{\partial \sigma}{\partial \Omega}\right)_{T D S C}^{F}=\left(\frac{\partial \sigma}{\partial \Omega}\right)_{T D S C}^{90^{\circ}}
$$

if pump beam polarization $\perp$ to scattered light direction

This TDSC is measured without polarizer, in the $90^{\circ}$ configuration with the pump beam laser linearly polarized in the direction perpendicular to the plane defined by the pump beam and the detected scattered light for TDCS measurements. It is thus the sum of the two orthogonal polarization components. In the forward direction, the largest part of the scattered intensity is polarized along the pump beam polarization. If subscript $z$ (respectively $x$ ) stands for the polarized component along the pump polarization (respectively orthogonal to it), then, with straightforward notations, the total TDSC can be decomposed as:

$$
\left(\frac{\partial \sigma}{\partial \Omega}\right)_{T D S C}^{F, T o t}=\left(1+\rho_{S}\right)\left(\frac{\partial \sigma}{\partial \Omega}\right)_{T D S C}^{F, z}
$$

with the depolarization ratio,

$$
\rho_{S}=\left(\frac{\partial \sigma}{\partial \Omega}\right)_{T D S C}^{F, x} /\left(\frac{\partial \sigma}{\partial \Omega}\right)_{T D S C}^{F, z}
$$

As explained in reference [32], this depolarization ratio does not depend on the angle of observation. Its value measured in the $90^{\circ}$ configuration is valid in the forward direction. It is also tabulated in the literature, so that, given the reported value for the $90^{\circ}$ TDSC (equal to the forward TDSC) and given the value for $\rho_{S}$, we deduce the value of the TDSC of interest for our polarization and forward direction from Eq. (A3). Inserting this value in Eq. (A1) gives the value for the Raman coefficient we are interested in. The correction brought by taking into account the depolarization ratio is quite often relatively small. However for some lines and liquids, it brings a major correction to the TDSC [32].

Taking all these corrections into account, the expression of the "parallel" Raman coefficient experienced by a forward Stokes beam in a nanofibre is expressed by:

$$
g_{R}=\frac{1}{1+\rho_{S}} \frac{8 \pi}{\Delta v_{F W H M}} \frac{N c^{2}}{\omega_{S}^{3} \hbar n_{S}^{2}}\left(\frac{\partial \sigma}{\partial \Omega}\right)_{T D S C}^{90^{\circ}}
$$

The Raman scattering cross-section, taken here at the maximum of the Raman line, is itself wavelength dependent. An approximate formula for this dependence is:

$$
\left(\frac{\partial \sigma}{\partial \Omega}\right)_{T D S C}=\frac{A \omega_{S}^{4}}{\left(\omega_{i}^{2}-\omega_{P}^{2}\right)^{2}}
$$

with $A$ a constant of proportionality, $\omega_{P}$ the pump angular frequency and the angular frequency of a material resonance, located in the UV domain. If $\omega_{P}$ is negligible compared to $\omega_{i}$ then, from Eq. (A1) and A6, we see that the Raman coefficient scales with the Stokes frequency.

\subsection{Raman coefficients of various liquids}

For many liquids, the parameters allowing to compute the Raman coefficients from Eq. (A5) and (A6) can be found in the literature. As explained above, we are only interested in the main Raman line. In the following we first present the references in which these parameters can be found. We then summarized in a table the results for these liquids.

\subsubsection{References for methanol}

The refractive index is given in [33]. Various values for the linewidth, cross-section and depolarization ratio have been published in [35], which corrects other values previously published in [34]. The uncertainty on the TDSC is about $\pm 10 \%$ [34].

\subsubsection{References for ethanol}

The refractive index dependence is from [36]. The other values are extracted from the same references as for methanol [34, 35].

\subsubsection{References for benzene}

The wavelength and temperature dependence of the refractive indices of benzene and of its derivative has been recently measured [37]. The Raman coefficient of benzene is also calculated taking into account the cross-section from [34]. The depolarization ratio is from [38]. Indeed, the value published in [32] is probably overestimated as it is integrated over the whole Raman line while the depolarization ratio is minimum at the peak wavelength [38].

\subsubsection{References for toluene}

As for benzene, we refer to [37] for the wavelength dependence of the refractive index of toluene. We rely on the Raman 


\begin{tabular}{|c|c|c|c|c|c|}
\hline & $\begin{array}{c}\text { Refractive } \\
\text { index at } \lambda_{P}\end{array}$ & $\begin{array}{c}\text { Raman coeff. } \\
\text { for } 1^{\text {st }} \text { Stokes } \\
\left(10^{-11} \mathrm{~m} \cdot \mathrm{W}^{-1}\right)\end{array}$ & $\begin{array}{c}1^{\text {st }} \text { Stokes } \\
\text { order } \lambda_{S 1}(\mathrm{~nm})\end{array}$ & $\begin{array}{c}\text { Raman coeff. } \\
\text { for } 2^{\text {nd }} \text { Stokes } \\
\left(10^{-11}{\left.\mathrm{~m} . \mathrm{W}^{-1}\right)}\right.\end{array}$ & $\begin{array}{c}2^{\text {nd }} \text { Stokes } \\
\text { order } \lambda_{S 2}(\mathrm{~nm})\end{array}$ \\
\hline Methanol & 1.34 & 0.213 & 627 & 0.175 & 761 \\
\hline Ethanol & 1.36 & 0.291 & 630 & 0.237 & 773 \\
\hline Benzene & 1.50 & 5.48 & 562 & 5.17 & 595 \\
\hline Toluene & 1.50 & 3.47 & 562 & 3.28 & 621 \\
\hline Nitrobenzene & 1.55 & 3.64 & 573 & 3.36 & 621 \\
\hline
\end{tabular}

TABLE A1 Refractive indices and Raman coefficients of a few liquids. The pump is assumed to be at $532 \mathrm{~nm}$.

cross-section from [39] measured at $488 \mathrm{~nm}$. The same reference also gives a Raman cross-section of benzene that is in perfect agreement with the one quoted above. The only measurement of the depolarization ratio we found is given in [40]. It is equal to 0.06 . The same reference also gives a depolarization ratio for benzene equal to 0.06 , which is larger than later reported values, typically around 0.02 . These values thus seem to be overestimated. We therefore take the same value for toluene as for benzene: 0.02 . We use the linewidth given in [41].

\subsubsection{References for nitrobenzene}

We have not found in the literature any function for approximating the dispersion of the refractive index of nitrobenzene. However according to [42] the refractive index dispersion in the visible is relatively flat so that we use the approximate value for the visible $n=1.55$. As for benzene and toluene, the value of the Raman cross-section is reported in [35]. Within the uncertainty, this value is consistent with the value reported in [25]. This same reference [25] also reports the value of the depolarization ratio. We use the linewidth given in [26].

\subsubsection{Summary of the main computed values}

We have gathered in the following table the values of the Raman coefficient of the above liquids when pumped at the wavelength of $\lambda_{P}=532 \mathrm{~nm}$. We consider the cascade of Raman effects, that is the pump being converted in a first Stokes order at $\lambda_{S 1}$, this first Stokes order being then itself converted in a second Stokes order at $\lambda_{S 2}$ and so on. Therefore, in the following table, we also give the values of the Raman coefficient at $\lambda_{S 1}$ giving rise to $\lambda_{S 2}$.

\subsection{Binary mixture of liquids}

\subsubsection{Refractive index}

In order to preserve the guiding properties of the nanofibre, the refractive index of the surrounding liquid has to be kept below the one of the silica core. We mix the Raman liquids whose refractive indices, $n_{A}$, are larger than the one of silica, such as benzene or toluene, with a low refractive index liquid (such as methanol or ethanol) whose index is noted $n_{B}$. Provided that these two liquids are miscible with no volume change, then the refractive index of the mixture $n_{\text {Mixt }}$ can be easily computed. Various formulae are listed in the literature. In our work, we use the Lorentz-Lorentz equation that is the most commonly used and one of the most accurate $[45,46]$ :

$$
\frac{n_{\text {Mixt }}^{2}-1}{n_{\text {Mixt }}^{2}+2}=\phi_{A} \frac{n_{A}^{2}-1}{n_{A}^{2}+2}+\phi_{B} \frac{n_{B}^{2}-1}{n_{B}^{2}+2}
$$

with $\phi_{A}$ and $\phi_{B}$ are the fraction volume of the liquids $\mathrm{A}$ and B.

\subsubsection{Raman coefficient}

According to Eq. (A1), the Raman coefficient has two dependences with the fraction volume $\phi_{A}$ of compound $\mathrm{A}$. The first lies in the dependence of the refractive index with $\phi_{A}$. The other dependence, for non-interacting mixtures, stems from the Raman coefficient proportionality to the number of molecules per unit volume. It is thus also proportional to the volume fraction. A hidden variation with the refractive index is also found in the value of the cross-section. Indeed, the cross-section of a molecule depends on the local optical electric fields at the pump and Stokes frequencies $F_{P}$ and $F_{S}$ $[47,48]$ :

$$
\left(\frac{\partial \sigma}{\partial \Omega}\right)_{T D S C}=\frac{n_{S}}{n_{P}}\left(F_{S} F_{P}\right)^{2}\left(\frac{\partial \sigma}{\partial \Omega}\right)_{T D S C}^{G a s}
$$

where $(\partial \sigma / \partial \Omega)_{\text {TDSC }}^{\text {Gas }}$ stands for the Raman coefficient in the gaseous phase and $n_{P}$ and $n_{S}$ the refractive indices at the pump and Stokes frequencies.

Using the Lorentz model, these local field factors are expressed as:

$$
F_{P, S} \approx \frac{2+n_{P, S}^{2^{2}}}{3}
$$

Taking into account all the dependences, we take the Raman coefficient $g_{R}\left(\phi_{A}\right)$ of compound $\mathrm{A}$ in the mixture as being:

$$
\begin{gathered}
g_{R}\left(\phi_{A}\right)=g_{R, A} \phi_{A} c\left(\phi_{A}\right) \text { with } \\
c\left(\phi_{A}\right)=\frac{\left(F_{S}\left(\phi_{A}\right) F_{P}\left(\phi_{A}\right)\right)^{2}}{\left(F_{S}(1) F_{P}(1)\right)^{2}} \frac{n_{A, S} n_{A, P}}{n_{M i x t, S}\left(\phi_{A}\right) n_{M i x t, P}\left(\phi_{A}\right)}
\end{gathered}
$$

with $g_{R, A}$ the Raman coefficient of compound $\mathrm{A}$ if taken alone and $F_{P, S}(1)$ the local field factors for the pure liquid A.

\section{References}

[1] L. M. Tong, J. Y. Lou, and E. Mazur, "Single-mode guiding properties of subwavelength-diameter silica and silicon wire waveguides," Opt. Express 12, 1025-1035 (2004). 
[2] L. M. Tong, F. Zi, X. Guo, and J. Y. Lou, "Optical microfibers and nanofibers: A tutorial," Opt. Commun. 285, 464-4647 (2012).

[3] M. A. Foster, A. C. Turner, M. Lipson, and A. L. Gaeta, “Nonlinear optics in photonic nanowires," Opt. Express 16, 1300-1320 (2008).

[4] T. A. Birks, W. J. Wadsworth, and P. St. J. Russell, "Supercontinuum generation in tapered fibers," Opt. Lett. 25, 1415-1417 (2000).

[5] J. M. Harbold, F. Ö. Ilday, F. W. Wise, T. A. Birks, W. J. Wadsworth, and $Z$. Chen, "Long-wavelength continuum generation about the second dispersion zero of a tapered fiber," Opt. Lett. 27, 1558-1560 (2002).

[6] R. Zhang, J. Teipel, X. Zhang, D. Nau, and H. Giessen, "Group velocity dispersion of tapered fibers immersed in different liquids," Opt. Express 12, 1700-1707 (2004).

[7] S. Leon-Saval, T. Birks, W. Wadsworth, P. St. J. Russell, and M. Mason, "Supercontinuum generation in submicron fiber waveguides," Opt. Express 12, 2864-2869 (2004).

[8] C. M. B. Cordeiro, W. J. Wadsworth, T. A. Birks, and P. St. J. Russell, "Engineering the dispersion of tapered fibers for supercontinuum generation with a $1064 \mathrm{~nm}$ pump laser," Opt. Lett. 30, 1980-1982 (2005).

[9] R. R. Gattass, G. T. Svacha, L. M. Tong, and E. Mazur, "Supercontinuum generation in submicrometer diameter silica fibers," Opt. Express 14, 9408-9414 (2006).

[10] D. D. Hudson, E. C. Mägli, A. C. Judge, S. A. Dekker, and B. J. Eggleton, "Highly nonlinear chalcogenide glass micro/nanofiber devices: Design, theory, and octave-spanning spectral generation," Opt. Commun. 285, 4660-4669 (2012).

[11] S. Richard, "Second-harmonic generation in tapered optical fibers," J. Opt. Soc. Am. B 27, 1504-1512 (2010).

[12] A. Couillet, and Ph. Grelu, "Third-harmonic generation in optical microfibers: From silica experiments to highly nonlinear glass prospects," Opt. Commun. 285, 3493-3497 (2012).

[13] R. Ismaeel, T. Lee, M. Ding, N. G. R. Broderick, and G. Brambilla, "Nonlinear microfiber loop resonators for resonantly enhanced third harmonic generation," Opt. Lett. 37, 5121-5123 (2012).

[14] J. Y. Lou, L. M. Tong, and Z. Z. Ye, "Modeling of silica nanowires for optical sensing," Opt. Express 13, 2135-2140 (2005).

[15] K. P. Nayak, P. N. Melentiev, M. Morinaga, Fam Le Kien, V. I. Balykin, and K. Hakuta, "Optical nanofiber as an efficient tool for manipulating and probing atomic Fluorescence," Opt. Express 15, 5431-5438 (2007).

[16] X. Guo, and L. M. Tong, "Supported microfiber loops for optical sensing," Opt. Express 16, 14429-14434 (2008).

[17] S. W. Harun, K. S. Lim, S. S. A. Damanhuri, and H. Ahmad, "Microfiber loop resonator based temperature sensor," J. Europ. Opt. Soc. Rap. Public. 6, 11026 (2011).

[18] L. Shan, G. Pauliat, L. M. Tong, and S. Lebrun “Optimal nanofiber dimensions for stimulated Raman scattering in the evanescent field," in Proceedings to the European Optical Society Annual Meeting (EOS, Aberdeen, 2012).

[19] L. Shan, G. Pauliat, L. M. Tong, and S. Lebrun "Demonstration of stimulated Raman scattering in the evanescent field of a tapered nanofiber," in Proceedings to the European Optical Society Annual Meeting (EOS, Aberdeen, 2012).

[20] R. Ismaeel, T. Lee, M. Ding, M. Belal, and G. Brambilla, "Optical microfiber passive components," Laser Photonics Rev., 1-35 (2012).

[21] G. Agrawal, Nonlinear Fiber Optics (Academic Press, London, 2007).
[22] A. W. Snyder, and J. Love, Optical Waveguide Theory (Kluwer Academic Publishers, London, 1983).

[23] J. Bures, Optique Guidée : fibres optiques et composants passifs tout-fibre (Presses internationales Polytechnique, Montreal, 2009).

[24] M. D. Turner, T. M. Monro, and S. Afshar, "A full vectorial model for pulse propagation in emerging waveguides with subwavelength structures part II: Stimulated Raman Scattering," Opt. Express 17, 11565-11581 (2009).

[25] R. H. Stolen, Clinton Lee, and R. K. Jain, "Development of the stimulated Raman spectrum in single-mode silica fibers," J. Opt. Soc. Am. B 1, 652-657 (1984).

[26] R. G. Smith, "Optical Power Handling Capacity of Low Loss Optical Fibers as Determined by Stimulated Raman and Brillouin Scattering," Appl. Opt 11, 2489-2494 (1972).

[27] E. Landahl, D. Baiocchi, and J. R. Thomson, "A simple analytic model for noise shaping by an optical fiber Raman generator," Opt. Commun. 150, 339-347 (1998).

[28] S. Yiou, P. Delaye, A. Rouvie, J. Chinaud, R. Frey, G. Roosen, P. Viale, et al., "Stimulated Raman scattering in an ethanol core microstructured optical fiber," Opt. Express 13, 4786-4791 (2005).

[29] S. Lebrun, P. Delaye, R. Frey, and G. Roosen, “High-efficiency single-mode Raman generation in a liquid-filled photonic bandgap fiber," Opt. Lett. 32, 337-339 (2007).

[30] S. Lebrun, C. Buy, P. Delaye, R. Frey, G. Pauliat, and G. Roosen, "Optical characterizations of a Raman generator based on a hollow core photonic crystal fiber filled with a liquid," J. Nonlinear 0pt. Phys. It Mat. 19, 101-109 (2009).

[31] M. Maier, W. Kaiser, and J. A. Giordmaine, "Backward Stimulated Raman Scattering," Phys. Rev. 177, 580-599 (1969).

[32] S. P. S. Porto, "Angular Dependence and Depolarization Ratio of the Raman Effect," J. Opt. Soc. Am. 56, 1585-1589 (1966).

[33] H. El-Kashef, "The necessary requirements imposed on polar dielectric laser dye solvents," Physica B: Condensed Matter 279, 295-301 (2000).

[34] M. J. Colles, and J. E. Griffiths, "Relative and absolute Raman scattering cross section in liquids," J. Chem. Phys. 56, 3384-3391 (1971).

[35] J. E. Griffiths, "Raman-scattering cross-sections in strongly interacting liquid-systems - $\mathrm{CH}_{3} \mathrm{OH}, \mathrm{C}_{2} \mathrm{H}_{5} \mathrm{OH}, \mathrm{I}-\mathrm{C}_{3} \mathrm{H}_{7} \mathrm{OH},\left(\mathrm{CH}_{3}\right)_{2} \mathrm{CO}, \mathrm{H}_{2} \mathrm{O}$, and $\mathrm{D}_{2} \mathrm{O}$," J. Chem Phys. 60, 2556 (1974).

[36] J. Rheims, J. Köser, and T. Wriedt, "Refractive-index measurements in the near-IR using an Abbe refractometer," Meas. Sci. Technol. 8 , 601-605 (1997).

[37] J. E. F. Rubio, J. M. Arsuaga, M. Taravillo, V. G. Baonza, and M. Caceres, "Refractive index of benzene and methyl derivatives: temperature and wavelength dependencies," Exp. Therm. Fluid. Sci. 28, 887-891 (2004).

[38] W. Proffitt, and S. P. S. Porto, "Depolarization ratio in Raman spectroscopy as a function of frequency," J. 0pt. Soc. Am. 63, 77-80 (1973).

[39] Y. Kato, and H. Takuma, "Absolute Measurement of RamanScattering Cross Sections of Liquids," J. Opt. Soc. Am. 61, 347-350 (1971).

[40] F. J. McClung, and D. Weiner, "Measurement of Raman Scattering Cross Sections for Use in Calculating Stimulated Raman Scattering Effects," J. Opt. Soc. Am. 54, 641-641 (1964). 
[41] W. R. L. Clements, and B. P. Stoicheff, "Raman linewidths for stimulated threshold and gain calculations," Appl. Phys. Lett. 12, 246-248 (1968).

[42] V. G. Foster, "Determination of the refractive index dispersion of liquid nitrobenzene in the visible and ultraviolet," J. Phys. D: Appl. Phys. 25, 525-529 (1992).

[43] K. Sakamoto, G. Mizutani, and S. Ushioda, "Absolute Ramanscattering cross section of a surface-adsorbed layer: Amorphous nitrobenzene on Ni(111)," Phys. Rev. B 48, 8993-9005 (1993).

[44] J.G. Skinner, and W. G. Nilsen, "Absolute Raman Scattering CrossSection Measurement of the $992 \mathrm{~cm}^{-1}$ Line of Benzene," J. Opt. Soc. Am. 58, 113-118 (1968).

[45] K. Narendra, P. Narayanamurthy, and Ch. Srinivasu, "Refractive Indices of Binary Liquid Mixture at Different Temperatures," Asian Journal of Applied Sciences 4, 535-541 (2011).
[46] R. Mehra, "Application of refractive index mixing rules in binary systems of hexadecane and heptadecane with n-alkanols at different temperatures," Proceedings of the Indian Academy of Sciences-Chemical Sciences 115, 147-154 (2003).

[47] J. R. Armstrong, N. Bloembergen, J. Ducuing, and P. S. Pershan, "Interaction between light waves in a nonlinear dielectric," Phys. Rev. 127, 1918-1939 (1962).

[48] P. D. Maker, and R. W. Terhune, "Study of Optical Effects Due to an Induced Polarization Third Order in the Electric Field Strength," Phys. Rev. 137, A801 (1965).

and errata, P. D. Maker, and R. W. Terhune, "Study of Optical Effects Due to an Induced Polarization Third Order in the Electric Field Strength," Phys. Rev. A 148, 990-990 (1966). 